\title{
Student understanding of graph slope and area under a graph: A comparison of physics and nonphysics students
}

\author{
Ana Susac, ${ }^{1,2, *}$ Andreja Bubic, ${ }^{3}$ Elizabeta Kazotti, ${ }^{2}$ Maja Planinic, ${ }^{2}$ and Marijan Palmovic ${ }^{4}$ \\ ${ }^{1}$ Department of Applied Physics, Faculty of Electrical Engineering and Computing, \\ University of Zagreb, Unska 3, 10000 Zagreb, Croatia \\ ${ }^{2}$ Department of Physics, Faculty of Science, University of Zagreb, Bijenicka 32, 10000 Zagreb, Croatia \\ ${ }^{3}$ Chair for Psychology, Faculty of Humanities and Social Sciences, \\ University of Split, Sinjska 2, 21000 Split, Croatia \\ ${ }^{4}$ Laboratory for Psycholinguistic Research, Department of Speech and Language Pathology, \\ University of Zagreb, Borongajska cesta 83h, 10000 Zagreb, Croatia
}

(Received 19 April 2018; published 18 September 2018)

\begin{abstract}
Previous studies have identified physics students' difficulties with graph slope and area under a graph in different contexts. In this study we compared physics and nonphysics (psychology) students' understanding of graphs; i.e., we evaluated the effects of concept (graph slope vs area under graph), type of question (qualitative vs quantitative), and context (physics vs finance) on their scores, strategies, and eye-tracking data. All students solved questions about graph slope better than the questions about area under a graph. Psychology students scored rather low on the questions about area under a graph, and physics students spent more time on questions about area under a graph than on slope questions, indicating that understanding of area under a graph is quite a difficult concept that seems unlikely to develop spontaneously. Generally, physics students had comparable scores on the qualitative and quantitative questions, whereas psychology students solved qualitative questions much better. As expected, students' scores and eye-tracking measures indicated that problems involving physics context were easier for physics students since they typically had higher scores and shorter total and axes viewing times for physics than finance questions. Some physics students may have transferred the concepts and techniques from physics to finance because they typically scored better than psychology students on the finance questions that were novel for both groups. Analysis of student strategies showed that physics students mostly relied on strategies learned in physics courses, with strong emphasis on the use of formulas, whereas psychology students mostly used common-sense strategies, as they did not know the physics formulas. The implications of the results for teaching and learning about graphs in physics courses are also discussed.
\end{abstract}

DOI: 10.1103/PhysRevPhysEducRes.14.020109

\section{INTRODUCTION}

Reading and interpreting information from graphs is an important skill in science and mathematics, but also in everyday life, because information in newspapers, on the Internet and TV is often conveyed through graphs. Student understanding of graphs has been investigated in many physics education research (PER) studies [1-23], but

\footnotetext{
* Corresponding author.

Department of Applied Physics, Faculty of Electrical Engineering and Computing, University of Zagreb, Unska 3, 10000 Zagreb, Croatia.

ana.susac@fer.hr

Published by the American Physical Society under the terms of the Creative Commons Attribution 4.0 International license. Further distribution of this work must maintain attribution to the author(s) and the published article's title, journal citation, and DOI.
}

sometimes also researchers in mathematics education [24-30] and educational psychology [31-36] used kinematics graphs to explore student graphing abilities.

The first PER studies on graphs identified student difficulties in connecting graphs to physical concepts in kinematics and to the real world [1]. McDermott et al. reported five dominant student difficulties in connecting graphs to physical concepts: discriminating between the slope and height of a graph, interpreting changes in height and changes in slope, relating one type of graph to another, matching narrative information with relevant features of a graph, and interpreting the area under a graph [1]. They have also found the following student difficulties in connecting graphs to the real world: difficulty representing continuous motion by a continuous line, separating the shape of a graph from the path of the motion, representing negative velocity on a $v$ vs $t$ graph, representing constant acceleration on an $a$ vs $t$ graph, and distinguishing among different types of motion graphs. In addition to these 
student difficulties with graphs, Leinhardt et al. reported the interval-point confusion that refers to the situations when students incorrectly focus on a single point although a range of points (an interval) is more appropriate, e.g., when comparing the growth of two populations [25]. Similar conceptual difficulties have been observed among high school and university students enrolled in both algebra-based and calculus-based introductory physics courses, and among in-service physics teachers [1].

Simultaneously with the research on student difficulties with graphs, PER researchers developed microcomputerbased labs and investigated how real-time graphing helps students in developing a better understanding of kinematics graphs $[2,4,13,17,18,21,23]$. In 1994, Beichner [19] developed the Test of Understanding of Graphs in Kinematics (TUG-K), which became one of the most widely used PER assessment instruments that was recently modified by Zavala et al. [15]. More recently, a number of studies investigated and compared university students' understanding of graphs in mathematics, physics, and other contexts [7-12,14]. Most studies explored student interpretation of graph slope while there are only a few studies on student understanding of area under a graph $[5,6,10,11,14,15]$.

Physics teachers often assume that the main cause of student difficulties with graphs lies in their difficulties with mathematical concepts related to graphs, such as the graph slope [8]. Two PER studies including questions without physical context on comparing the values of the integral of the two functions shown over the same interval and on comparing of the graph slopes confirmed that students have difficulties with the math concepts [5,7]. However, our studies with parallel (isomorphic) problems in mathematics, physics, and other contexts have shown that parallel problems with added context (physics or other context) were more difficult than the corresponding mathematics problems $[8,10,11,14]$. This suggested student difficulties with transfer of knowledge between mathematics and physics (or other contexts). Analogous results were obtained in the studies by Wemyss and van Kampen [9] and Bollen et al. [12]. They found that students solved questions on water level vs time graphs better than the corresponding questions on distance vs time graphs, although they had never encountered former graphs in the formal educational setting. The analysis of students' responses and the categorization of their strategies revealed that they used similar correct and incorrect strategies regardless of country (Ireland, Belgium, and Spain in Ref. [12], Croatia and Austria in Ref. [14]) or the level of mathematical proficiency (algebra-based or calculus-based physics courses); only the prevalence of certain strategies sometimes varied.

All previous studies were conducted on physics students, so we decided to additionally explore nonphysics students' understanding of graphs in both physics and everyday contexts. For everyday context we have chosen the finance context that all students are familiar with. We expected that nonphysics students would not be able to apply physics formulas, which is the dominant strategy of physics students, so they would use more intuitive strategies. Thus, we could infer from the comparison of their strategies what may be intuitive and what students have to learn formally about graph slope and area under a graph.

In addition to students' scores and explanations on problems with graphs that we analyzed in the previous studies $[10,11,14]$, we used eye tracking in this study to investigate where students allocate visual attention during problem solving. Measurement of eye movements is an increasingly used method in PER [37-49]. There are a number of eye-tracking studies on understanding of graphs $[36,39,46,48-51]$. In some studies kinematics graphs were used to investigate graph comprehension [36,39,46,48,49], otherwise different graphs with everyday contexts were employed [50,51].

Carpenter and Shah used line graphs of three continuous variables that can be shown in two different ways and allow different levels of complexity [50]. Their results suggested that even the simple graphs require rather complex cognitive processes. The authors proposed a model of the comprehension of line graphs that integrates perceptual and conceptual processes. Perceptual processes refer to pattern-recognition processes, such as the encoding and interpreting of the slopes and patterns of the line graphs. Conceptual processes refer to the interpreting of the observed patterns, such as the interpreting of labels and scales. Integration of these two processes in an iterative process is the basis of graph understanding.

Goldberg and Helfman used eye tracking to compare reading values on linear and radial graphs [51]. Their results indicated that linear graphs more efficiently conveyed information than radial graphs. Mapping a data point to its value was faster on linear than radial graphs because their horizontally or vertically aligned labels are clearer compared to radial graphs. Based on their results, the authors have proposed guidelines for designing radial and linear graphs. Although that one and similar studies may be informative for the PER community, they do not give an insight into student understanding of the more specific concepts related to graphs, such as graph slope and area under a graph, which are required in physics teaching and learning.

As already mentioned, there are several eye-tracking studies in which kinematics graphs were used. Kozhevnikov et al. examined how students' spatial visualization ability is related to their problems-solving skills [36]. Among the different types of kinematics problems, their study participants solved one problem with a kinematics graph. It was found that the low-spatial students more often interpreted the graph as a literal representation of an object's motion compared to high-spatial students. Further analyses of eye-tracking data revealed that the highspatial students spent longer time viewing the axes than the low-spatial students. The authors concluded that the spatial 
visualization ability has an important role in solving kinematics problems with spatial parameters.

Furthermore, an eye-tracking study conducted by Madsen et al. [43] on differences between those who correctly and incorrectly answer physics problems has shown that students who answered correctly spent longer time looking at the relevant areas of the graph. This indicated that the previous experience and expertise helped students to guide their visual attention during problem solving. Three of the six problems used in this study were on kinematics graphs. In the follow-up study only one kinematics graph problem was used (with three other problems) to examine the effects of visual cueing on students' eye movements and reasoning during problem solving [44]. Although visual cues helped participants to better solve some problems and demonstrate more expertlike pattern of eye movements, they did not influence their solving of the kinematics graph problem.

Similarly, Kekule used six problems from the previous studies on graphs $[1,19]$ to investigate differences in eye movements between the best and the worst performing students $[46,48]$. She found that the best performers had shorter total fixation duration for each task than the worst performing students. Similar results were found for a number of fixations, whereas no statistically significant difference between the best and the worst performing students was found for fixation duration. In this comparison, only four students were included in each group. In the following studies conducted in the Czech Republic and Finland [39,49] a larger number of students participated, and the representational variant of the Force Concept Inventory was used [52]. The results showed that graph representation was easier than the motion map representation, probably because students are more familiar with that graph representation. Analogous to the previous studies, students who did not solve problems correctly paid more attention to the irrelevant and more visually salient parts of problem.

Overall, eye tracking was used in several studies in which participants were solving problems with graphs, but usually only a small number of problems was used and/or a small number of students participated. Student understanding of important concepts related to graphs, such as graph slope and area under a graph, was usually not in the focus of these studies. Thus, we decided to use eye tracking to explore student understanding of graph slope and area under a graph in addition to a paper-and-pencil test.

\section{RESEARCH QUESTIONS}

The main research question of this study was "What are the differences between physics and nonphysics students in solving problems with graphs?". More specifically, the aim of this study was to evaluate the effects of the following:

(a) concept (graph slope vs area under a graph),

(b) type of question (qualitative vs quantitative),

(c) question context (physics vs finance) on students' scores, their problem-solving strategies, and eye-tracking data.

\section{METHODS}

\section{A. Participants}

Participants in this study were 90 undergraduate students from the University of Zagreb, Croatia. Half of the participants $(N=45)$ were prospective physics teachers, whereas the other half of the participants were psychology majors. Most of the participants were fourth-year students, and their average age was $23 \pm 2$ years. Each participant gave an informed written consent before taking part in the experiment.

Physics is taught as a compulsory subject in the last two grades of all elementary schools and throughout four years of most of high schools in Croatia. Pupils are taught kinematics graphs at the age 15 and 16 (last grade of elementary school and first year of high school). Psychology students were not exposed to the teaching on kinematics graphs after high school, while physics students learned about kinematics graphs also in several university courses. Physics and psychology students had not encountered graphs related to prices, money, etc., in their formal education.

\section{B. Materials}

Eight multiple-choice test items were developed or modified from our previous study [8]. All problems with graphs used in the study are given in the Supplemental Material [53]. Four sets of isomorphic questions about graphs in the context of physics (kinematics) and finance referred to the qualitative and quantitative understanding of the graph slope and area under a graph. Isomorphic questions required the same mathematical procedure in the context of kinematics graphs and graphs related to prices (we will refer to them as finance graphs). The text of the question and the appearance of the graph were similar for the isomorphic test items, to enable the comparison of the effects of the two contexts. We prepared two versions of the test, with a counterbalanced sequence of questions from physics and finance. The isomorphic questions were never presented one after another.

In a short questionnaire, participants were asked after the test if they had noticed any similarity between the questions, how they solved the problems with graphs, what the interpretation of the slope and the area under a $v$ vs $t$ graph was, to write the formula for acceleration, how the slope of a line graph is calculated, and to write the formula for the area of a right-angled triangle.

\section{Apparatus}

Eye-movement data were recorded using a stationary eye-tracking system with a temporal resolution of $500 \mathrm{~Hz}$ and a spatial resolution of $0.25^{\circ}-0.50^{\circ}$ (SMI iView HiSpeed system, Senso Motoric Instruments G.m.b.H.). The distance between the eyes and the monitor was $50 \mathrm{~cm}$. 
Prior to every recording, the gaze of each participant was calibrated with a 13-point calibration algorithm. The gaze direction was calculated as a vector between corneal reflection (which is stable, i.e., it depends only on head movements) and pupil position (i.e., the calculated center of the pupil). A fixation can be defined as the state when the eye remains still over a period of time, while a saccade is the rapid motion of the eye from one fixation to another. Smaller eye movements that occur during fixations, such as tremors, drifts, and flicks are called microsaccades. Microsaccades were automatically grouped in a fixation. The fixations were detected automatically using the "Event Detected Method," which is built into the eye-tracking device. Blinks were corrected automatically.

\section{Procedure}

First, the participants were familiarized with the apparatus and the way to answer the questions (by pressing the enter key on the keyboard, and by choosing the answer using the mouse). The participants were asked to keep their head fixed during the measurements, so they could not use paper and pencil. After calibration, questions were presented to a participant one by one. By choosing the answer, the participant advanced to the next question. There was no time limit to answer the questions. The whole procedure, including preparation, eye-movement calibration and recording, lasted around $20 \mathrm{~min}$.

After the measurement of eye movements, students solved the same questions using a paper-and-pencil test and giving explanations for their answers. They also answered a short questionnaire on similarity between questions, their strategy in solving problems with graphs, and basic facts about graph slope and area under a graph.

\section{E. Data analysis}

The test was scored independently by two authors. The agreement between the raters was very high (96\%) and the differences in scoring have been discussed and consensually resolved. The maximum score was 8 points. If a correct answer during the eye-tracking measurement was given with a correct explanation in the paper-and-pencil test, the student was awarded 1 point. If a correct answer was given with a wrong explanation, the student was awarded 0 points. A correct answer without correct explanation indicated that the correct answer was probably selected by chance or for a wrong reason. This happened more often for psychology students (15.8\% of all questions) than for physics students (6.4\% of all questions).

Recorded eye-movement data were analyzed using BeGaze software that calculated the viewing time (dwell time) for any defined area of interest (AOI). AOIs were rectangles that included introduction text and question (Question), graph (Graph), and multiple-choice answers (Multiple choice) as shown on Fig. 1. AOI All included all these AOIs and were used for determining the total viewing

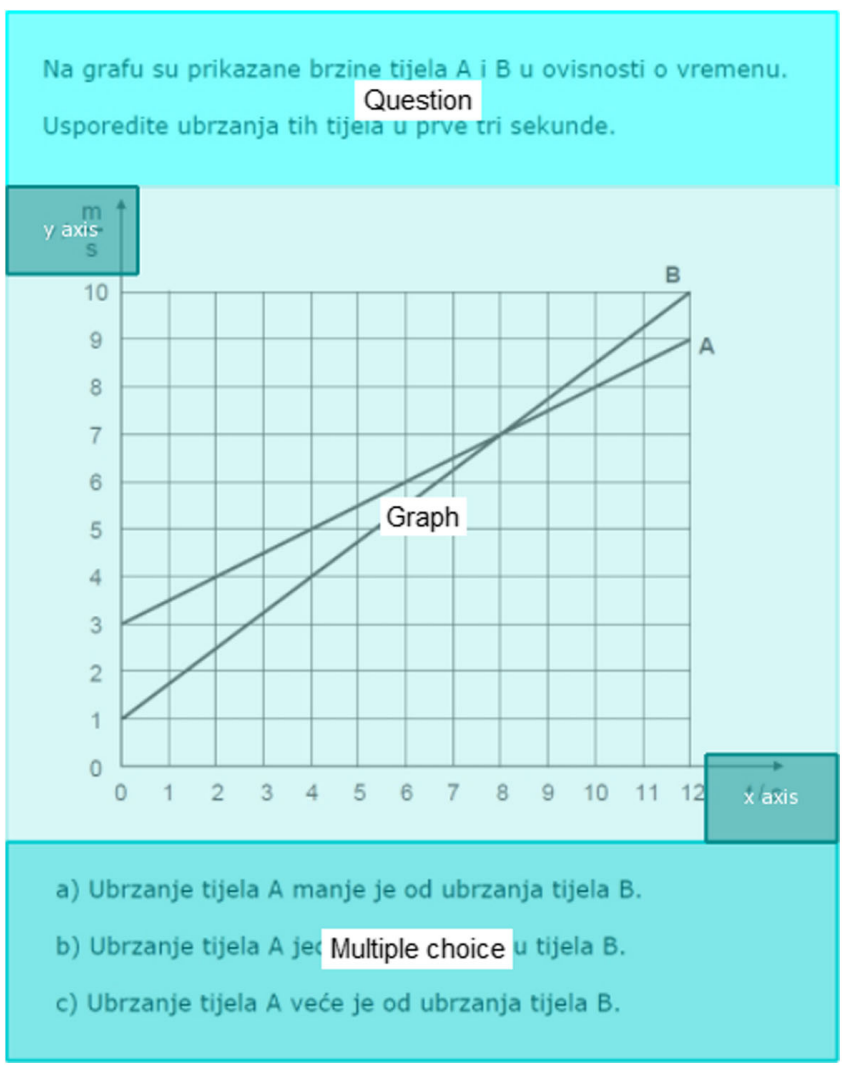

FIG. 1. Example of definition of AOIs for one test item.

time. In addition, AOIs' $x$ axis and $y$ axis were defined to include axes labels and units as illustrated in Fig. 1. The axes labels' viewing time was calculated as the sum of the viewing time (dwell time) for the AOIs' $x$ axis and $y$ axis.

To determine the effects of the type of question (qualitative vs quantitative), concept (graph slope vs area under a graph), group (physics students vs psychology students) and context (physics vs finance) on student scores and viewing time, several two-way analyses of variance (ANOVAs) and Bonferroni corrected student's $t$ tests were conducted. The data that were analyzed using ANOVAs, and are reported in this manuscript, satisfied the assumptions required for conducting ANOVAs. Distributions of data and residuals were normal, and the homogeneity of variance was tested using Levene's test that was not significant. A threshold of $p=$ 0.05 was used for determining the level of effect significance within all conducted tests. The Bonferroni correction was used to avoid false-positive results (type I errors) when multiple pairwise tests were performed after ANOVA. This correction adjusts probability $(\boldsymbol{p})$ values by dividing the critical $\boldsymbol{p}$ value by the number of comparisons being made.

\section{RESULTS}

\section{A. Analysis of students' scores}

The mean test score and standard deviation were $(50 \pm 28) \%$. The score of physics students $(68 \pm 25) \%$ 
(a)

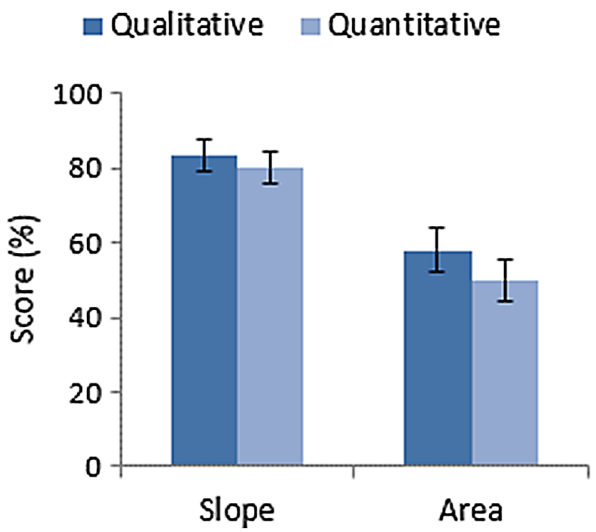

(b)

Psychology students

$\square$ Qualitative $\square$ Quantitative

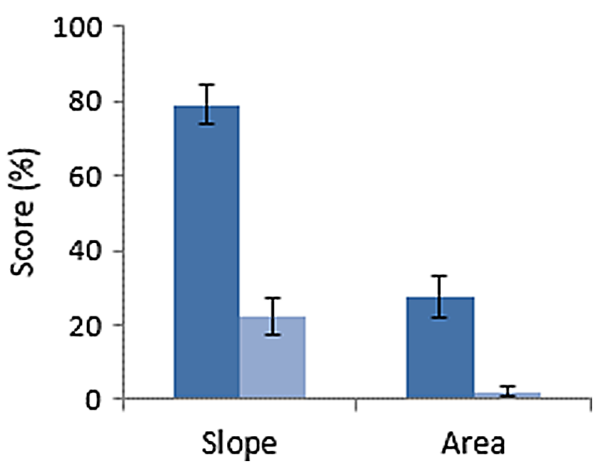

FIG. 2. (a) Average scores of physics students on the qualitative and quantitative questions about graph slope and area under a graph. (b) Average scores of psychology students on the qualitative and quantitative questions about graph slope and area under a graph. The error bars represent 1 standard error of the mean (SEM).

was higher than the score of psychology students $(33 \pm 19) \%(p<0.0001)$.

To compare the students' scores on qualitative and quantitative questions about graph slope and area under a graph, we conducted a two-way repeated-measures ANOVA with factors of type of question (qualitative vs quantitative) and concept (graph slope vs area under a graph), separately for physics and psychology students. For physics students, a significant main effect of concept $\left[F(1,44)=36.78, p<0.0001, \eta_{p}{ }^{2}=0.455\right]$ was found, whereas the effect of type of question $[F(1,44)=2.05$, $\left.p>0.05, \eta_{p}{ }^{2}=0.044\right]$ and interaction effect $[F(1,44)=$ $\left.0.34, p>0.05, \eta_{p}{ }^{2}=0.008\right]$ were not significant. Physics students had higher scores on questions about graph slope than on questions about area under a graph [Fig. 2(a)].

For psychology students, the results showed a statistically significant main effect of both factors, type of question $\left[F(1,44)=75.55, p<0.0001, \eta_{p}{ }^{2}=0.632\right]$ and concept $\left[F(1,44)=98.65, p<0.0001, \eta_{p}{ }^{2}=0.691\right]$, as well as their interaction $[F(1,44)=12.25, p=0.001$, $\left.\eta_{p}{ }^{2}=0.218\right]$. Psychology students had higher scores on qualitative questions than on quantitative questions, and they scored higher on questions about graph slope compared to questions about area under a graph [Fig. 2(b)].
The difference between scores on qualitative and quantitative questions was larger for questions about graph slope.

To further explore student scores, for each pair of isomorphic questions we performed a two-way mixeddesign ANOVA with between-subjects factor group (physics students vs psychology students) and within-subjects factor context (physics vs finance). The results are shown in Table I. For qualitative questions on slope, only the interaction of factors was statistically significant indicating that for psychology students the finance graphs were easier than the kinematics graphs $[t(44)=2.46, p<0.05]$ while there was the opposite trend for physics students, but the difference was not statistically significant (Fig. 3). For quantitative questions on slope, physics students had higher scores than psychology students and students' scores on the physics question were higher compared to the finance question. In particular, physics students solved better the physics question than the finance question $[t(44)=2.88, p<0.05]$.

For qualitative questions on area, physics students had higher scores than psychology students. They solved the physics question better than the finance question $[t(44)=$ 2.66, $p<0.05$ ], while there was the opposite trend for psychology students, but the difference was not statistically significant (Fig. 3). For quantitative questions on area,

TABLE I. Results of the two-way ANOVAs conducted on students' scores on qualitative and quantitative questions about graph slope and area under a graph with factors group (physics students vs psychology students) and context (physics vs finance).

\begin{tabular}{|c|c|c|c|c|c|c|c|c|c|}
\hline & \multicolumn{3}{|c|}{ Group } & \multicolumn{3}{|c|}{ Context } & \multicolumn{3}{|c|}{ Interaction } \\
\hline & $F$ & $p$ & $\eta_{p}^{2}$ & $F$ & $p$ & $\eta_{p}{ }^{2}$ & $F$ & $p$ & $\eta_{p}{ }^{2}$ \\
\hline Slope qualitative & 0.42 & $>0.05$ & 0.005 & 0.94 & $>0.05$ & 0.011 & 5.85 & 0.018 & 0.062 \\
\hline Slope quantitative & 77.97 & $<10^{-4}$ & 0.470 & 8.18 & 0.005 & 0.085 & 1.50 & $>0.05$ & 0.017 \\
\hline Area qualitative & 13.44 & $<10^{-4}$ & 0.133 & 1.01 & $>0.05$ & 0.011 & 9.08 & 0.003 & 0.094 \\
\hline Area quantitative & 69.77 & $<10^{-4}$ & 0.442 & 11.49 & 0.001 & 0.115 & 6.18 & 0.015 & 0.066 \\
\hline
\end{tabular}




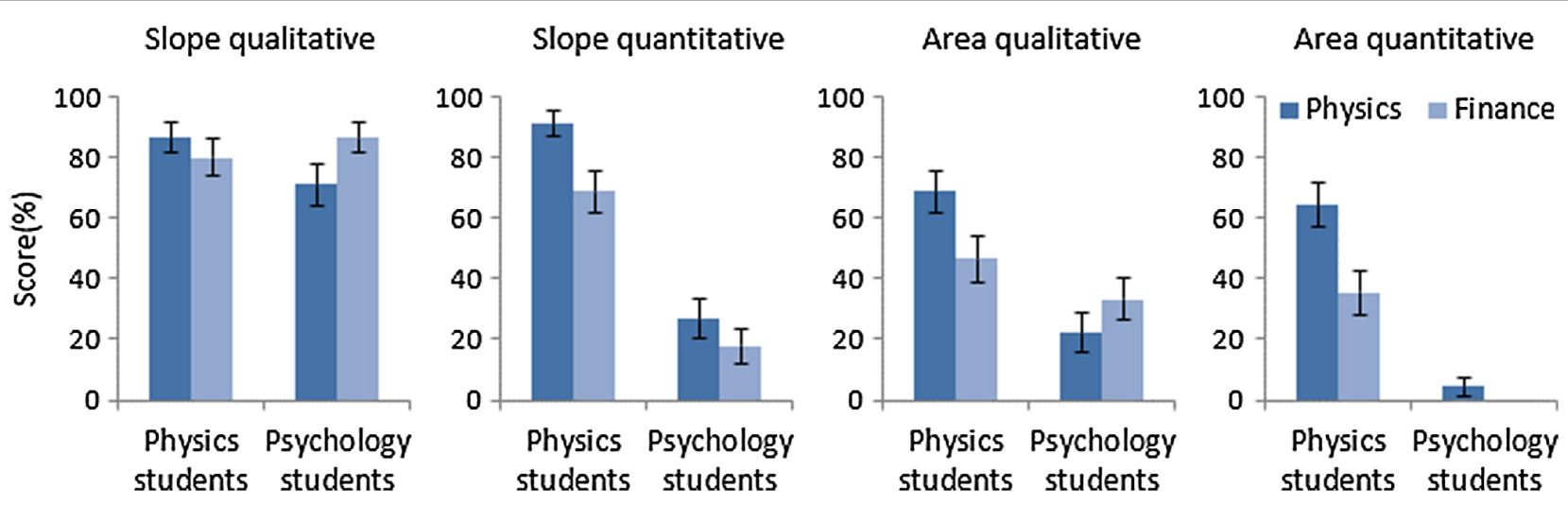

FIG. 3. Average scores of physics and psychology students in the context of physics and finance on the qualitative and quantitative questions about graph slope and area under a graph. The error bars represent 1 SEM.

physics students again had higher scores than psychology students, and they better solved the physics question than the finance question $[t(44)=3.10, p<0.01]$. On these questions, psychology students had very low scores; only two students solved physics question (Fig. 3).

\section{B. Analysis of eye-tracking data}

First, we analyzed total viewing time, i.e., dwell time that students spent attending each question before giving the answer. To compare the students' total viewing time on qualitative and quantitative questions about graph slope and area under a graph, we conducted a two-way repeatedmeasures ANOVA with factors type of question (qualitative vs quantitative) and concept (graph slope vs area under a graph), separately for physics and psychology students. For physics students, a significant main effect of concept $\left[F(1,44)=38.71, p<0.0001, \eta_{p}{ }^{2}=0.468\right]$ was found, whereas the effect of the type of question was not significant $\left[F(1,44)=0.35, \quad p>0.05, \quad \eta_{p}{ }^{2}=0.008\right]$. Physics students spent less time viewing the questions

(a)

Physics students

Q Qualitative $\square$ Quantitative

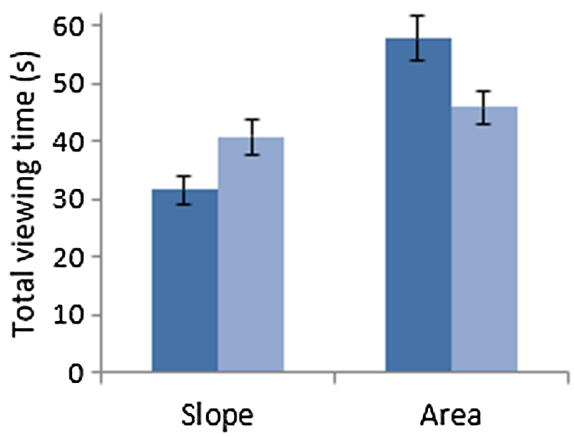

about graph slope than questions about area under a graph [Fig. 4(a)]. The interaction effect was significant $\left[F(1,44)=27.13, p<0.0001, \eta_{p}{ }^{2}=0.381\right]$ indicating that type of question (qualitative vs quantitative) had the opposite effect on the total time viewing time on questions about graph slope and questions about area under a graph. On questions about slope, physics students had a longer total viewing time for the quantitative question $[t(44)=$ 2.86, $p<0.05]$, whereas they had longer total viewing time for the qualitative question about area under a graph $[t(44)=3.70, p<0.01]$.

For psychology students, the results were analogous; the main effect of the type of question was statistically significant $\left[F(1,44)=9.89, \quad p=0.003, \quad \eta_{p}{ }^{2}=0.184\right]$ while the main effect of concept was not statistically significant $\left[F(1,44)=1.04, \quad p>0.05, \quad \eta_{p}{ }^{2}=0.023\right]$. Psychology students spent less time viewing the questions about graph slope than the questions about area under a graph [Fig. 4(b)]. The interaction effect of the factors type of question and concept was statistically significant

(b)

Psychology students

a Qualitative $\quad$ Quantitative

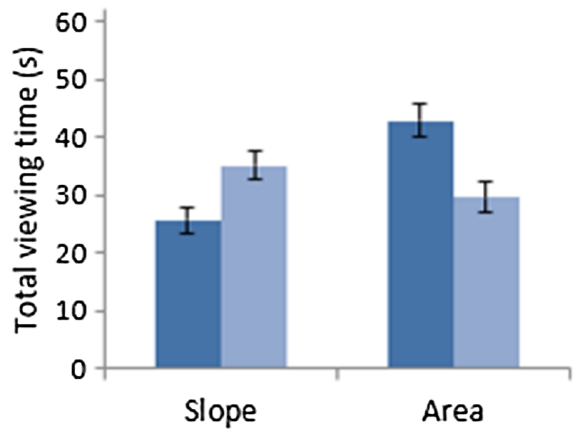

FIG. 4. (a) Average total viewing time of physics students on the qualitative and quantitative questions about graph slope and area under a graph. (b) Average total viewing time of psychology students on the qualitative and quantitative questions about graph slope and area under a graph. The error bars represent 1 SEM. 
TABLE II. Results of the two-way ANOVAs conducted on students' total viewing times on qualitative and quantitative questions about graph slope and area under a graph with factors group (physics students vs psychology students) and factors context (physics vs finance).

\begin{tabular}{|c|c|c|c|c|c|c|c|c|c|}
\hline & \multicolumn{3}{|c|}{ Group } & \multicolumn{3}{|c|}{ Context } & \multicolumn{3}{|c|}{ Interaction } \\
\hline & $F$ & $p$ & $\eta_{p}{ }^{2}$ & $F$ & $p$ & $\eta_{p}{ }^{2}$ & $F$ & $p$ & $\eta_{p}^{2}$ \\
\hline Slope qualitative & 3.36 & $>0.05$ & 0.037 & 0.03 & $>0.05$ & $<10^{-4}$ & 1.26 & $>0.05$ & 0.014 \\
\hline Slope quantitative & 1.96 & $>0.05$ & 0.022 & 5.68 & 0.019 & 0.061 & 1.42 & $>0.05$ & 0.016 \\
\hline Area qualitative & 9.80 & 0.002 & 0.100 & 1.41 & $>0.05$ & 0.016 & 0.82 & $>0.05$ & 0.009 \\
\hline Area quantitative & 16.98 & $<10^{-4}$ & 0.162 & 11.95 & 0.001 & 0.120 & 1.64 & $>0.05$ & 0.018 \\
\hline
\end{tabular}

$\left[F(1,44)=56.73, p<0.0001, \eta_{p}^{2}=0.563\right]$. Similar to physics students, psychology students had longer total viewing time for the quantitative question about slope $[t(44)=5.39, p<0.0001]$, whereas they had had longer total viewing time for the qualitative question about area under a graph $[t(44)=4.72, p<0.0001]$.

Analogous to the analysis of student scores, we performed a two-way mixed-design ANOVA with betweensubjects factor group (physics students vs psychology students) and within-subjects factor context (physics vs finance) on total viewing time for each pair of isomorphic questions. The results are shown in Table II. For qualitative questions on slope, there was no difference between physics and psychology students, and the context also did not affect the total viewing time (Fig. 5). For quantitative questions on slope, students had longer total viewing time for the finance question compared to the physics question. Physics students had longer total viewing time on both pairs of isomorphic questions on area under a graph. In addition, on quantitative questions about area under a graph, students had longer total viewing time for the finance question (Fig. 5).

To further investigate the eye-tracking data, we compared the viewing time on different areas of interest (AOIs) for physics and psychology students (Fig. 6). A Bonferroni adjusted $t$ test showed that physics students looked longer at the AOI Graph than psychology students $[t(88)=3.74$, $p<0.0001]$. Although there was a similar trend that physics students looked longer at the AOI Question compared to psychology students, the difference was not statistically significant after Bonferroni correction $[t(88)=$ $2.31, p=0.069]$. There was also no statistical difference between the viewing time of physics and psychology students at the AOI Multiple choice $[t(88)=1.30$, $p>0.05]$.

As previous studies reported an important role of graph axes in understanding graphs [36], we analyzed viewing time on the AOI Axes that was composed of the AOIs $x$ axis and $y$ axis (Fig. 1). We performed a two-way mixed-design ANOVA with between-subjects factor group (physics students vs psychology students) and within-subjects factor context (physics vs finance) on viewing time on the AOI Axes for each pair of isomorphic questions (Fig. 7 in the Appendix). The results are shown in Table $\mathrm{V}$ in the Appendix. For qualitative questions on slope, there were no statistically significant effects. For other three pairs of isomorphic questions (slope quantitative, area qualitative, and area quantitative), the interaction of factors group and context was statistically significant. For questions on area under a graph, physics students spent more time viewing the axes on the finance graphs compared to the physics graphs $[t(44)=2.40, p<0.05$ for the qualitative questions on area; $t(44)=3.48, p<0.05$ for the quantitative questions on area]. A similar trend was observed for the
Slope qualitative

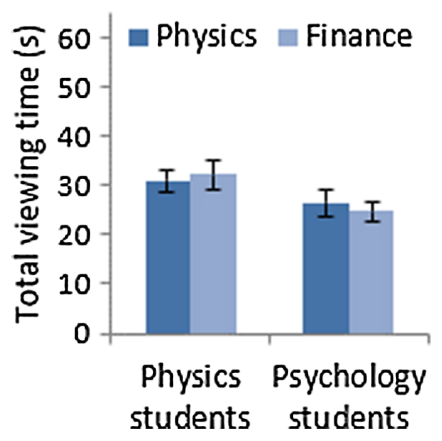

Slope quantitative

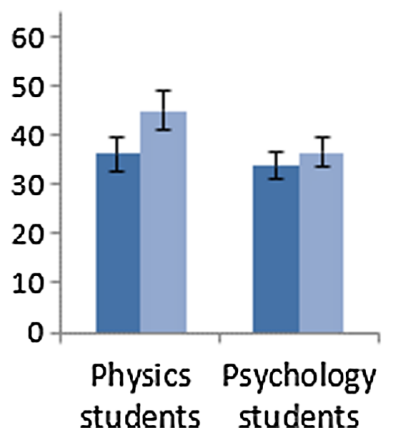

Area qualitative

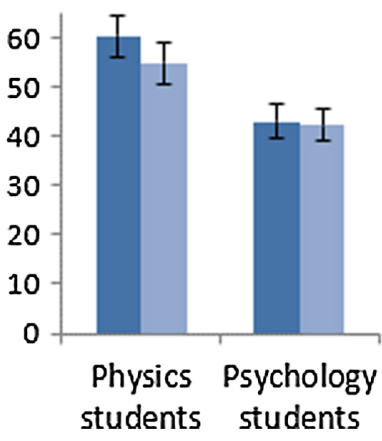

Area quantitative

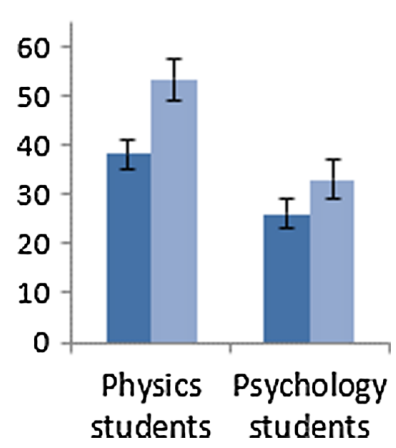

FIG. 5. Average total viewing time of physics and psychology students in the context of physics and finance on the qualitative and quantitative questions about graph slope and area under a graph. The error bars represent 1 SEM. 
— Physics students $=$ Psychology students

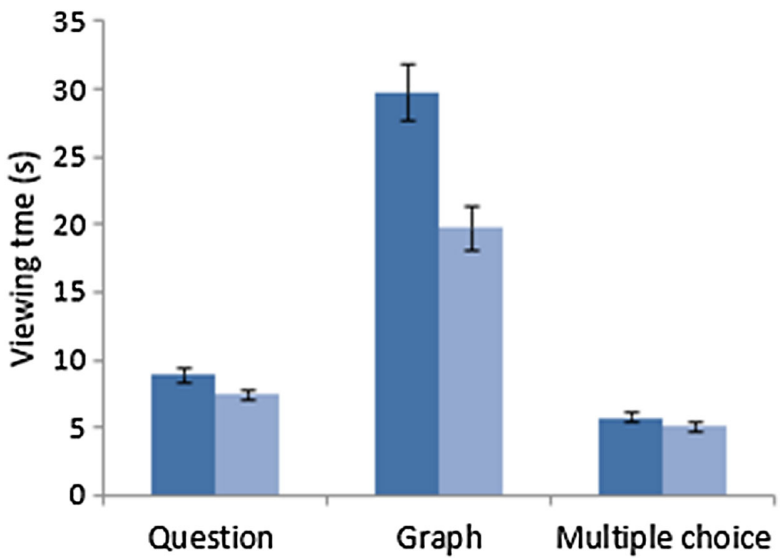

FIG. 6. Average viewing time of physics and psychology students on the AOIs Question, Graph, and Multiple choice. The error bars represent 1 SEM.

quantitative questions on slope, but the difference was not statistically significant after Bonferroni correction $[t(44)=2.09, p=0.08]$.

\section{Analysis of student strategies}

To compare physics and psychology students' reasoning about graphs, we identified their strategies through the analysis of their procedures and explanations provided in the paper-and-pencil test. The overviews of students' strategies on questions about graph slope and area under a graph are given in Tables III and IV.

On the qualitative questions about graph slope, most students used the terms "slope" and "steepness" in their explanations. It is interesting that 54\% of psychology students (who used this strategy) used the term steepness, $28 \%$ of them used the term slope, and the remaining $18 \%$ used "steepness of slope" in their explanation. Almost all physics students $(97 \%)$ used the term slope. However, on the question "How do you calculate the slope of a line graph?" in the questionnaire, 10 physics students (out of 45) did not answer correctly, and they mostly exchanged the numerator and the denominator in the correct expression $\Delta y / \Delta x$. Only 3 (out of 45) psychology students responded accurately to this question, but $44 \%$ of them knew that acceleration is the slope of the $v$ vs $t$ graph.

Physics students mostly used formulas, especially on the quantitative questions about graph slope. Although they relied on physics formulas, six physics students (out of 45) did not write the correct formula for acceleration in in the questionnaire; they wrote $a=v / t$ instead of $a=\Delta v / \Delta t$. Psychology students used formulas very rarely and only a small number of them (6 out of 45) remembered even the incorrect formula for acceleration $(a=v / t)$. Although it is possible that some students did not write deltas in the formula for acceleration as a form of sloppiness or laziness, the formula $a=v / t$ usually refers to the strategy of determining the acceleration on an $a$ vs $t$ graph from one point only, instead of choosing two points and calculating the slope of a graph.

The most frequently used correct strategy by psychology students on the quantitative questions about graph slope was reasoning based on rise over run (Table III) that represents some sort of a common-sense strategy. The psychology students also often used that strategy on the qualitative questions. Physics students mostly used reasoning based on rise over run on the quantitative finance question.

Although the questions on slope were rather well solved, some students reasoned incorrectly or did not have any strategy; e.g., more than $20 \%$ of psychology students did not write any explanation or procedure when they gave the incorrect answer on the quantitative question about graph slope. The most common incorrect strategy was reasoning based on interval-point confusion. A fraction of psychology students showed interval-point confusion on all questions about slope while a few physics students reasoned

TABLE III. An overview of strategies used by students for the slope questions. The left numbers show percentages of physics students and the right numbers percentages of psychology students who used a particular strategy.

\begin{tabular}{|c|c|c|c|c|}
\hline Correct strategy & $\begin{array}{c}\text { Physics } \\
\text { qualitative/\% }\end{array}$ & $\begin{array}{c}\text { Finance } \\
\text { qualitative } / \%\end{array}$ & $\begin{array}{c}\text { Physics } \\
\text { quantitative/\% }\end{array}$ & $\begin{array}{c}\text { Finance } \\
\text { quantitative/\% }\end{array}$ \\
\hline Reasoning based on graph slope or steepness & $67 / 51$ & $71 / 51$ & $0 / 0$ & $0 / 0$ \\
\hline Reasoning based on rise over run & $13 / 27$ & $11 / 40$ & $18 / 29$ & $44 / 29$ \\
\hline Correct use of physics (or constructed) formulas & $13 / 0$ & $2 / 0$ & $73 / 4$ & $29 / 0$ \\
\hline Other strategies & $2 / 2$ & $4 / 2$ & $0 / 0$ & $0 / 0$ \\
\hline Noncategorizable or no explanation (or procedure) & $7 / 13$ & $7 / 9$ & $7 / 9$ & $9 / 4$ \\
\hline Incorrect strategy & $\begin{array}{c}\text { Physics } \\
\text { qualitative/\% }\end{array}$ & $\begin{array}{c}\text { Finance } \\
\text { qualitative/\% }\end{array}$ & $\begin{array}{c}\text { Physics } \\
\text { quantitative/\% }\end{array}$ & $\begin{array}{c}\text { Finance } \\
\text { quantitative/\% }\end{array}$ \\
\hline Reasoning based on interval-point confusion & $0 / 11$ & $0 / 7$ & $4 / 18$ & $16 / 36$ \\
\hline Other strategies & $0 / 9$ & $7 / 0$ & $2 / 9$ & $0 / 9$ \\
\hline Noncategorizable or no explanation (or procedure) & $0 / 0$ & $0 / 0$ & $0 / 27$ & $2 / 22$ \\
\hline
\end{tabular}


TABLE IV. An overview of strategies used by students for the area questions. The left numbers show percentages of physics students and the right numbers percentages of psychology students who used a particular strategy.

\begin{tabular}{|c|c|c|c|c|}
\hline Correct strategy & $\begin{array}{c}\text { Physics } \\
\text { qualitative/\% }\end{array}$ & $\begin{array}{c}\text { Finance } \\
\text { qualitative } / \%\end{array}$ & $\begin{array}{c}\text { Physics } \\
\text { quantitative/\% }\end{array}$ & $\begin{array}{c}\text { Finance } \\
\text { quantitative/\% }\end{array}$ \\
\hline Comparison or calculation of area under the graph & $56 / 2$ & $38 / 7$ & $51 / 4$ & $38 / 7$ \\
\hline $\begin{array}{l}\text { Reasoning based on "larger velocity or price for longer } \\
\text { time" idea }\end{array}$ & $7 / 13$ & $11 / 22$ & $0 / 0$ & $0 / 0$ \\
\hline Correct use of physics formulas & $9 / 0$ & $0 / 0$ & $20 / 0$ & $4 / 0$ \\
\hline Other strategies & $9 / 9$ & $2 / 7$ & $0 / 4$ & $0 / 0$ \\
\hline Noncategorizable or no explanation (or procedure) & $2 / 4$ & $9 / 7$ & $0 / 4$ & $2 / 0$ \\
\hline Incorrect strategy & $\begin{array}{l}\text { Physics } \\
\text { qualitative/\% }\end{array}$ & $\begin{array}{l}\text { Finance } \\
\text { qualitative/\% }\end{array}$ & $\begin{array}{l}\text { Physics } \\
\text { quantitative/\% }\end{array}$ & $\begin{array}{l}\text { Finance } \\
\text { quantitative/\% }\end{array}$ \\
\hline $\begin{array}{l}\text { Reading off initial and/or final } y \text { coordinate value } \\
\text { from the graph }\end{array}$ & $7 / 16$ & $16 / 27$ & $0 / 40$ & $13 / 40$ \\
\hline Reading off both coordinate values and multiplication $(x y)$ & $2 / 0$ & $0 / 0$ & $2 / 24$ & $24 / 38$ \\
\hline Reasoning based on graph appearance (usually its slope) & $9 / 18$ & $13 / 18$ & $0 / 0$ & $0 / 0$ \\
\hline Use of inappropriate physics formula $s=v t$ & $0 / 11$ & $0 / 0$ & $20 / 11$ & $0 / 0$ \\
\hline $\begin{array}{l}\text { Reasoning based on graph as an actual picture of the } \\
\text { motion }\end{array}$ & $0 / 11$ & $0 / 0$ & $0 / 0$ & $0 / 0$ \\
\hline Other strategies & $2 / 2$ & $2 / 7$ & $0 / 0$ & $9 / 4$ \\
\hline Noncategorizable or no explanation (or procedure) & $0 / 18$ & $11 / 13$ & $2 / 13$ & $9 / 11$ \\
\hline
\end{tabular}

incorrectly based on the interval-point confusion on the quantitative finance question. Other incorrect strategies were reasoning based on graph appearance and reasoning based on area under a graph instead of graph slope, but they were used by a small number of students.

Table IV shows that the most often used correct strategy on the questions about area under a graph was the comparison or calculation of area under the graph, and it was mostly used by physics students. This might lead to the conclusion that psychology students could not use this strategy because they did not know the meaning of the area under the $v$ vs $t$ graph. Nevertheless, the analysis of their responses in the questionnaire revealed that 18 psychology students (out of 45) knew that the area under the $v$ vs $t$ graph represents the covered distance. However, they neither applied this fact in solving problems related to the area under the $v$ vs $t$ graph, nor transferred this idea to the corresponding finance questions. In addition, the questionnaire showed that more than half of psychology students could not calculate the area of a right-angled triangle. Only 18 psychology students (out of 45) wrote the correct formula for the area of a right-angled triangle, whereas the remaining students mainly wrote some form (correct or incorrect) of the Pythagorean theorem that is not the appropriate formula for the area of a right-angled triangle.

The most frequently used correct reasoning strategy by psychology students on the qualitative questions about area under the graph was the common-sense reasoning strategy based on a "larger velocity or price for a longer time" idea, which was used to explain the larger total value of the required quantity. An example of the corresponding student's explanation is the following: "the time interval in which STAR is more expensive is longer than the one when the GOLD is more expensive, so finally, ride with the STAR is more expensive". This strategy is correct because both price per kilometer and velocity were given as linear functions, but it might not be correct if more complex functions were used. Psychology students used this strategy more often than physics students.

Only physics students used formulas on physics questions about the area under the graph. Psychology students could not use the formulas as they generally did not know them. Other correct strategies were related to calculation or estimation of average velocity or average price.

The most common incorrect strategy was reading off the initial and/or the final $y$ coordinate value from the graph. It was more often used by psychology students, especially on the quantitative questions about area under a graph. Psychology students also often used the strategy of reading off both coordinate values and multiplying them on the quantitative questions. A similar strategy was using the inappropriate physics formula $s=v t$. It was used by both psychology students on physics questions and physics students on the quantitative physics question. On the qualitative questions, some students used incorrect reasoning based on the graph appearance (usually its slope). On the qualitative physics question some psychology students considered graph as an actual picture of the motion. As for the questions on graph slope, psychology students, more often than the physics students, did not provide any explanation or procedure.

On the question "Have you noticed any similarity between questions you have solved?", most students answered that all questions had graphs, and some of them (27\% of physics students and $16 \%$ of psychology students) have also noticed the repetition of questions, but in a 
different context; a few students even identified some pairs of isomorphic questions. Other students answered that all questions are solved by using graph slope or area under a graph (20\% of physics students), or that they have noticed similarity in the graph appearance, such as the linearity of intersection of two lines. On the question "How do you solve problems with graphs?", students gave diverse answers; some of them (27\% of physics students and $20 \%$ of psychology students) mentioned that they looked at the axes, other answered that they read off values, determined relationships between the variables, observed slope, etc.

\section{DISCUSSION}

\section{A. Students' scores}

\section{Physics students understand graphs better than psychology students}

As expected, physics students had much higher scores than psychology students. However, our goal was to explore in more detail the scores, strategies, and attentional patterns of the physics and nonphysics students. This might bring some insight on what is intuitive and what students have to learn about graph slope and area under a graph.

\section{Graph slope is an easier concept than the area under a graph}

All students better solved the questions about graph slope than the questions about area under a graph. This corroborates our previous findings that interpreting the meaning of area under a graph is typically more difficult than interpreting the meaning of graph slope [10].

\section{Quantitative and qualitative questions are equally difficult for physics students}

The score of physics students on the qualitative and quantitative questions did not differ, whereas psychology students solved much better the qualitative than the quantitative questions. In our previous study [10] physics students better solved the qualitative than the quantitative questions on graph slope, but they were tested at the beginning of the first semester. Practically, according to their previous experience with graphs, they were similar to the nonphysics students in the present study. Physics students in this study were mostly fourth-year students, and their results suggest (when compared with the results of the first-year students from our previous study) that studying physics significantly improves their ability to solve quantitative problems on graphs. Although this may seem as an obvious result, it is relevant since teaching efforts unfortunately sometimes do not bring the expected outcomes. In this case, it seems that formal teaching and learning about graphs is efficient in developing quantitative reasoning skills.

\section{Slope is an intuitive idea}

On the qualitative questions on graph slope, average scores of the physics and psychology students did not differ. This may suggest that the idea of slope is quite intuitive, since psychology students who had not learned about kinematics graphs since the beginning of high school and have never learned about finance graphs solved these questions equally as well as physics students. Furthermore, it is interesting that psychology students better solved the qualitative question on slope in the finance context compared to physics (kinematics) context. This indicates that it is more difficult to recognize acceleration as the slope in the $v$ vs $t$ graph than to identify the growth rate of price as the slope in the price vs time graph. This is in agreement with the results from our previous study with similar questions on first-year university students [10], and studies which compared student responses to questions on distance vs time graphs with responses to isomorphic questions on linear water level vs time graphs $[9,12]$.

\section{Indications of transfer}

For all other pairs of isomorphic questions (slope quantitative, area qualitative, area quantitative), physics students had higher scores than psychology students. They also better solved the physics question than the finance question, which is not surprising, because they were exposed to kinematics graphs during their education. However, they solved rather well the finance question which they had probably never encountered before. This may indicate transfer, i.e., it seems that some physics students used strategies they developed in physics for solving finance problems.

\section{Some questions are too difficult for psychology students}

A rather small fraction of psychology students solved the quantitative questions about slope and the qualitative questions about area under a graph. Some of them probably remembered some kinematics concepts from high school, but it is interesting that they solved physics and finance questions equally well. The very low score of psychology students on quantitative questions about area under a graph indicate that these questions are unlikely to be solved intuitively, without formal learning of the topic.

\section{B. Eye-tracking data}

\section{Viewing time indicates cognitive load, i.e., question difficulty}

Generally, eye-tracking data give insight on how long students process information from the graph, thus providing an indirect measure of cognitive load. A longer viewing time indicates higher cognitive load $[54,55]$. Both physics and psychology students had similar total viewing times on the qualitative and quantitative questions about graph slope and area under a graph. All students typically spent more time solving questions about area under a graph, suggesting 
that this concept is more difficult for students than graph slope. This again confirms our previous results [10]. The reason for this might be that area under a graph is intrinsically a more difficult concept than graph slope, but it is also possible that students are less exposed to the concept of summation (integral) that is graphically represented by the area under a graph.

Furthermore, both physics and psychology students had a longer total viewing time for the quantitative question than for the qualitative question about slope. This indicates that the comparison of slopes in the qualitative question is a rather quick and intuitive process, while the calculation of slope in the quantitative question requires more time because relevant information has to be extracted from the graph. The opposite effect was observed for the question about area under a graph; students had a longer total viewing time for the qualitative question than for the quantitative question. This indicates that the comparison of areas under the graph is cognitively demanding, even if students (like many physics students) know what should be compared. Unlike the comparison of graph slopes, the comparison of areas under the graphs could not be easily grasped. On the other hand, the calculation of the area under a graph is probably not more demanding than the calculation of the slope (given that students recognize what should be calculated and know the appropriate formula).

The comparison between the physics and psychology students on the physics and finance questions revealed that physics students spent more time solving the questions about area under a graph. Although physics students are experts in graph solving compared to psychology students, it seems that they are also not as familiar with the concept of area under a graph as they are with the concept of graph slope [10]. Our results indicate that more emphasis should be put on teaching and learning the concept of area under a graph in introductory physics courses.

On the quantitative questions about slope and area under a graph, physics students spent more time solving the finance questions. This corroborates the above-mentioned results on physics students' scores and indicates that the new context of finance is more demanding than the context of kinematics that students are familiar with (especially physics students). The physics students had probably never encountered qualitative questions on area under a graph in both contexts and quantitative questions on area in the finance context, and on these questions, they had the longest total viewing time. It seems from our results that experts spend more time solving problems that they are not familiar with (but which are close to the area of their expertise) than nonexperts. Our results show that psychology students did not spend a very long time trying to solve the quantitative area questions suggesting that nonexperts may spend less time on problems that are very difficult for them (compared to experts). These results are the opposite of the previous reports on a shorter total fixation duration for the best performing students compared to the worst performing students [48]. It is possible that psychology students in our study did not perceive these problems as difficult, because of their reduced metacognitive insight [56], or that they perceived them as too difficult, and accordingly did not put too much effort into solving them. Additional studies are needed to further investigate the relationship between students' scores and the time spent on problems by experts and nonexperts.

\section{Physics students examine the graphs more carefully than psychology students}

The comparison of the viewing time on different AOIs revealed that physics students spent more time looking at the AOI Graph than psychology students. This indicates that physics students, although experts in comparison to psychology students, spent more time in extracting information from the graphs. This agrees with the previous studies reporting students' difficulties in graph processing $[1,9-12,14]$. It seems that psychology students (nonexperts for graphs) did not know where to look and what information to search for, so they spent less time viewing graphs.

The observed trend that physics students spent more time on the AOI Question than psychology students might indicate that physics students read more carefully the text of the question to identify which information they had to extract from the graph. In our previous eye-tracking study [41], we found that students spent longer time on the keywords in the text of the problem that helped them to extract the required information.

\section{Graph axes are important for understanding graphs}

When asked about their strategies in solving problems with graphs, some students mentioned that they looked at the axes. Kozhevnikov et al. also reported that the more successful students spent longer time viewing the axes while solving problem with the kinematics graph [36]. All this suggests that viewing axes is an important part of graph processing. The analysis of the viewing time on the AOI Axes revealed that physics students spent more time viewing the axes on the finance graphs than on the physics graphs in the questions about area under a graph. This indicates that they needed more time to extract relevant information from the axes in graphs with the context that was new for them. It is also possible that they spent more time on the axes titles in the finance graphs because they are longer (i.e., have more letters). However, that is probably not the case because a similar effect was not observed for psychology students. The significant interaction effect suggests that physics students usually spent less time on the axes in the physics graphs, whereas psychology students typically spent less time on the axes in the finance graphs. This shows that physics students are more familiar with the physics graphs while psychology students are more familiar with the finance context. Overall, these 
results confirm the important role of information contained in graph axes in understanding graphs. Students should be encouraged to explore graphs and especially to analyze graph axes.

\section{Student strategies}

Overall, the analysis of student strategies shows that psychology students more frequently used intuitive, common-sense strategies while physics students relied more on the use of formulas, especially in the familiar context (kinematics). For example, psychology students used reasoning based on rise over run more often than physics students. Physics students used this strategy more only in the quantitative finance question where they could not apply the known physics formulas. However, some of them constructed formulas in that question (Table I). Generally, students used the correct and incorrect strategies that were already identified in the previous studies [11,14].

\section{Student strategies on the slope questions}

Psychology students used more often the everyday word steepness than slope in their explanations, and some even used the term steepness of slope. This indicates that some of them probably do not have a clear idea of what the slope is. Nevertheless, even a vague idea of the steepness or slope of the graph allowed them to solve very well the qualitative questions on graph slope. Even some physics students do not completely understand the concept of graph slope; some of them do not know the mathematical formula for the slope of a line graph (as revealed in the questionnaire). This becomes evident on the quantitative question about a graphs' slope, because qualitative questions can be solved even with the imprecise idea of graph slope $[10,11,14]$.

The most common incorrect strategy on the slope questions was reasoning based on interval-point confusion. Psychology students employed this strategy more often than physics students. However, in an unfamiliar quantitative question about graph slope in the finance context, some physics students also used this strategy. More than $20 \%$ of psychology students did not provide any explanation or procedure when they gave an incorrect answer on the quantitative question about graphs slope. This indicates that they probably did not have any strategy (some of them even reported that in the questionnaire).

\section{Student strategies on the questions about area under a graph}

Furthermore, the analysis of student strategies on the questions on area under a graph revealed that physics students who correctly answered the questions mostly compared or calculated the area under the graph or used physics formulas. Psychology students could not rely on these strategies, as most of them did not know the correct formula for the area of a right-angled triangle or physics formulas. It is interesting that about $40 \%$ of psychology students remember that covered distance corresponds to the area under the $v$ vs $t$ graph. However, it seems that they remembered this as a learned fact because they were not able to apply it to the concrete problem.

Analogous to the questions on graph slopes, on the questions about area under a graph, psychology students used a common-sense strategy ("larger velocity or price for longer time") more often than physics students. This strategy was not reported before in our previous studies, because we did not have any qualitative questions on area under a graph $[10,11,14]$. In our previous study, we found that when students cannot rely on physics formulas, some of them come up with more creative strategies [11]. The results from this study corroborate the previous findings, because psychology students who did not know formulas mostly used common-sense strategies, such as the idea of a larger velocity or price for longer time.

In this study we identified similar incorrect strategies as in our previous study [11]: reading off initial and/or a final $y$ coordinate value from the graph, reading off both coordinate values and its multiplication, reasoning based on graph appearance (usually its slope), use of inappropriate physics formula and reasoning based on the graph as an actual picture of the motion. All these incorrect strategies were used by psychology students whereas physics students generally used them less often, but when they used them it was more often in the finance context. Psychology students, more often than physics students, did not provide any explanation or procedure indicating that they did not have any strategy in solving these questions.

\section{CONCLUSIONS}

The results of this study confirm our previous findings on student understanding of graph slope and area under a graph and bring some new insight into differences between graph experts (physics students) and nonexperts (psychology students).

All students solved the questions about graph slope better than the questions about the area under a graph. Psychology students had rather low scores on the questions about area under a graph, and physics students spent more time than psychology students on questions about area under a graph. These results indicate that area under a graph is quite a difficult concept that is unlikely to be developed without formal teaching and learning, and that more attention should be given to this topic in physics courses.

Physics and psychology students had comparable scores on the qualitative questions on slope which indicates that the idea of slope is rather intuitive. However, many psychology students were not able to calculate the slope, thus indicating that their idea of slope was rather vague. This suggests that the intuitive idea of slope, probably held by most students, should be further developed in physics 
courses and strongly linked to the mathematical concept of slope that enables students to quantify slope.

Generally, physics students solved the qualitative and the quantitative questions equally well, whereas psychology students solved qualitative questions much better than the quantitative questions. This is further evidence that learning physics helps students to develop deeper understanding of concepts and the ability to quantitatively express relationships between quantities.

As expected, students' scores and eye-tracking measures indicated that the physics context was easier for physics students. They typically had higher scores and shorter total and axes viewing times for the physics than the finance questions. However, the results suggested that some physics students may have transferred knowledge (concepts and techniques) from physics and mathematics to finance, a new context that they had not encountered before in an educational setting. The higher score of physics students compared to psychology students on the quantitative finance questions gave indirect evidence for the transfer of knowledge. Neither group of students was familiar with the finance questions, and it was probably the experience with calculations of slope and area under a graph in physics problems that helped physics students to apply the same techniques to the new finance context.

Analysis of student strategies showed that physics students mostly relied on strategies and techniques learned in the physics courses, with strong emphasis on the use of formulas. As psychology students mainly did not know formulas, they mostly used common-sense strategies. Certainly, it is good that physics students are familiar with formulas and prepared to use them while solving problems. However, sometimes the excessive reliance on formulas can be hindering because students fail to think about common-sense strategies that might be more useful in new contexts where no formulas are available. Nevertheless, some students are able to construct formulas for the new contexts, which is also an indication of transfer of knowledge in the sense of "preparation for future learning" [57]. Overall, students should be taught to use formulas in physics, and to apply the same techniques in other contexts, but they should also be encouraged to use other ways of reasoning in physics.

\section{ACKNOWLEDGMENTS}

This research was funded by the grant from the University of Zagreb Development Fund (198002).

\section{APPENDIX}

The average viewing time on the AOI Axes and the results of the two-way ANOVAs conducted to examine the effects of student group (physics students vs psychology students) and graph context (physics vs finance) are shown in Fig. 7 and Table V.
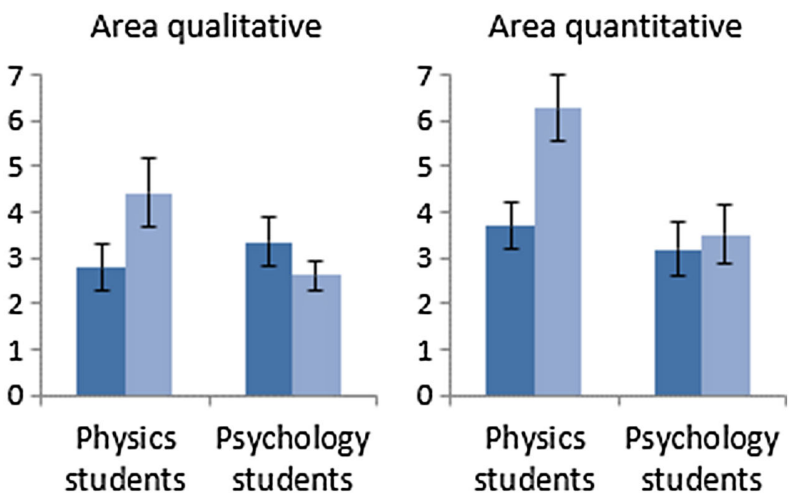

FIG. 7. Average viewing time on the AOI Axes of the physics and psychology students in the context of physics and finance on the qualitative and quantitative questions about graph slope and area under a graph. The error bars represent 1 SEM.

TABLE V. Results of the two-way ANOVAs conducted on the students' total viewing times on the qualitative and quantitative questions about graph slope and area under a graph with the between-subjects factor group (physics students vs psychology students) and within-subjects factor context (physics vs finance).

\begin{tabular}{|c|c|c|c|c|c|c|c|c|c|}
\hline & \multicolumn{3}{|c|}{ Group } & \multicolumn{3}{|c|}{ Context } & \multicolumn{3}{|c|}{ Interaction } \\
\hline & $F$ & $p$ & $\eta_{p}{ }^{2}$ & $F$ & $p$ & $\eta_{p}{ }^{2}$ & $F$ & $p$ & $\eta_{p}^{2}$ \\
\hline Slope qualitative & 0.02 & $>0.05$ & $<10^{-4}$ & 1.22 & $>0.05$ & 0.014 & 2.03 & $>0.05$ & $\overline{0.023}$ \\
\hline Slope quantitative & 2.32 & $>0.05$ & 0.026 & 0.06 & $>0.05$ & 0.001 & 6.36 & 0.013 & 0.067 \\
\hline Area qualitative & 0.97 & $>0.05$ & 0.011 & 1.16 & $>0.05$ & 0.013 & 8.28 & 0.005 & 0.086 \\
\hline Area quantitative & 5.42 & 0.022 & 0.058 & 7.51 & 0.007 & 0.079 & 4.60 & 0.035 & 0.050 \\
\hline
\end{tabular}


[1] L. C. McDermott, M. L. Rosenquist, and E. H. van Zee, Student difficulties in connecting graphs and physics: Examples from kinematics, Am. J. Phys. 55, 503 (1987).

[2] J. R. Mokros and R. F. Tinker, The impact of microcomputer-based labs on children's ability to interpret graphs, J. Res. Sci. Teach. 24, 369 (1987).

[3] P. A. Forster, Graphing in physics: Processes and sources of error in tertiary entrance examinations in Western Australia, Res. Sci. Educ. 34, 239 (2004).

[4] I. S. Araujo, E. A. Veit, and M. A. Moreira, Physics students' performance using computational modelling activities to improve kinematics graphs interpretation, Comput. Educ. 50, 1128 (2008).

[5] W. M. Christensen and J. R. Thompson, Investigating student understanding of physics concepts and the underlying calculus concepts in thermodynamics, in Proceedings of the 13th Annual Conference on Research in Undergraduate Mathematics Education (Mathematical Association of America, Oberlin, OH, 2010), Vol. 1.

[6] D. H. Nguyen and N. S. Rebello, Students' understanding and application of the area under the curve concept in physics problems, Phys. Rev. ST Phys. Educ. Res. 7, 010112 (2011).

[7] W. M. Christensen and J. R. Thompson, Investigating graphical representations of slope and derivative without a physics context, Phys. Rev. ST Phys. Educ. Res. 8, 023101 (2012).

[8] M. Planinic, Z. Milin-Sipus, H. Katic, A. Susac, and L. Ivanjek, Comparison of student understanding of line graph slope in physics and mathematics, Int. J. Sci. Math. Educ. 10, 1393 (2012).

[9] T. Wemyss and P. Van Kampen, Categorization of first-year university students' interpretations of numerical linear distance-time graphs, Phys. Rev. ST Phys. Educ. Res. 9, 010107 (2013).

[10] M. Planinic, L. Ivanjek, A. Susac, and Z. Milin-Sipus, Comparison of university students' understanding of graphs in different contexts, Phys. Rev. ST Phys. Educ. Res. 9, 020103 (2013).

[11] L. Ivanjek, A. Susac, M. Planinic, A. Andrasevic, and Z. Milin-Sipus, Student reasoning about graphs in different contexts, Phys. Rev. Phys. Educ. Res. 12, 010106 (2016).

[12] L. Bollen, M. De Cock, K. Zuza, J. Guisasola, and P. Van Kampen, Generalizing a categorization of students' interpretations of linear kinematics graphs, Phys. Rev. Phys. Educ. Res. 12, 010108 (2016).

[13] H. M. Brasell, The effect of real-time laboratory graphing on learning graphic representations of distance and velocity, J. Res. Sci. Teach. 24, 385 (1987).

[14] L. Ivanjek, M. Planinic, M. Hopf, and A. Susac, Student difficulties with graphs in different contexts, in Cognitive and Affective Aspects in Science Education Research, Contributions from Science Education Research, edited by K. Hahl, K. Juuti, J. Lampiselkä, A. Uitto, and J. Lavonen (Springer International Publishing, London, 2017), pp. 167-178, DOI: 10.1007/978-3-319-586854_13.
[15] G. Zavala, S. Tejeda, P. Barniol, and R. J. Beichner, Modifying the test of understanding graphs in kinematics, Phys. Rev. Phys. Educ. Res. 13, 020111 (2017).

[16] A.-M. Pendrill and L. Ouattara, Force, acceleration and velocity during trampoline jumps - a challenging assignment, Phys. Educ. 52, 065021 (2017).

[17] R. J. Beichner, The effect of simultaneous motion presentation and graph generation in a kinematics lab, J. Res. Sci. Teach. 27, 803 (1990).

[18] H. M. Brasell and M. B. Rowe, Graphing skills among high school physics students, School Sci. Math. 93, 63 (1993).

[19] R. J. Beichner, Testing student interpretation of kinematics graphs, Am. J. Phys. 62, 750 (1994).

[20] C. A. Berg and P. Smith, Assessing students' abilities to construct and interpret line graphs: Disparities between multiple-choice and free-response instruments, Sci. Educ. 78, 527 (1994).

[21] J. B. Brungardt and D. Zollman, The influence of interactive videodisk instruction using simultaneous time analysis on kinematic graphing skills of high school pshysics students, J. Res. Sci. Teach. 32, 855 (1995).

[22] J. Woolnough, How do students learn to apply their mathematical knowledge to interpret graphs in physics?, Res. Sci. Educ. 30, 259 (2000).

[23] I. Testa, G. Monroy, and E. Sassi, Students' reading images in kinematics: The case of real-time graphs, Int. J. Sci. Educ. 24, 235 (2002).

[24] D. Kerslake, Graphs, in Children's Understanding of Mathematics: 11-16, edited by K. M. Hart (John Murray, London, 1981), pp. 120-136.

[25] G. Leinhardt, O. Zaslavsky, and M. K. Stein, Functions, graphs, and graphing: Tasks, learning, and teaching, Rev. Educ. Res. 60, 1 (1990).

[26] A. A. DiSessa, D. Hammer, B. L. Sherin, and T. Kolpakowski, Inventing graphing: meta-representational expertise in children, J. Math. Behav. 10, 117 (1991); https://eric.ed.gov/?id=EJ443633.

[27] P. Swatton and R. M. Taylor, Pupil performance in graphical tasks and its relationship to the ability to handle variables, Br. Educ. Res. J. 20, 227 (1994).

[28] T. Graham and J. Sharp, An investigation into able students' understanding of motion graphs, Teach. Math. Its Appl. 18, 128 (1999).

[29] C. Hadjidemetriou and J. Williams, Children's graphical conceptions, Res. Math. Educ. 4, 69 (2002).

[30] I. Basson, Physics and mathematics as interrelated fields of thought development using acceleration as an example, Int. J. Math. Educ. Sci. Technol. 33, 679 (2002).

[31] M. C. Linn, J. W. Layman, and R. Nachmias, Cognitive consequences of microcomputer-based laboratories: Graphing skills development, Contemp. Educ. Psychol. 12, 244 (1987).

[32] R. Nemirovsky, C. Tierney, and T. Wright, Body motion and graphing, Cognit. Instr. 16, 119 (1998).

[33] M. Kozhevnikov, M. Hegarty, and R. Mayer, Students' use of imagery in solving qualitative problems in kinematics, ERIC Document Reproduction Service No. ED433239 (U.S. Department of Education, Washington, DC, 1999). 
[34] M. Kozhevnikov, M. Hegarty, and R. E. Mayer, Revising the visualizer- verbalizer dimension: evidence for two types of visualizers, Cognit. Instr. 20, 47 (2002).

[35] M. Kozhevnikov and R. Thornton, Real-time data display, spatial visualization ability, and learning force and motion concepts, J. Sci. Educ. Technol. 15, 111 (2006).

[36] M. Kozhevnikov, M. A. Motes, and M. Hegarty, Spatial visualization in physics problem solving, Cogn. Sci. 31, 549 (2007).

[37] T. van Gog, F. Paas, and J. J. G. Van Merriënboer, Uncovering expertise-related differences in troubleshooting performance: Combining eye movement and concurrent verbal protocol data, Appl. Cogn. Psychol. 19, 205 (2005).

[38] D. Rosengrant, A. Van Heuvelen, and E. Etkina, Do students use and understand free-body diagrams?, Phys. Rev. ST Phys. Educ. Res. 5, 010108 (2009).

[39] J. Viiri, M. Kekule, J. Isoniemi, and J. Hautala, Eyetracking the effects of representation on students' problem solving approaches, Proceedings of the Annual FMSERA Symposium 2016 (2017), pp. 88-98, https://journal.fi/ fmsera/article/view/60941.

[40] J. Han, L. Chen, Z. Fu, J. Fritchman, and L. Bao, Eye-tracking of visual attention in web-based assessment using the Force Concept Inventory, Eur. J. Phys. 38, 045702 (2017).

[41] A. Susac, A. Bubic, P. Martinjak, M. Planinic, and M. Palmovic, Graphical representations of data improve student understanding of measurement and uncertainty: An eye-tracking study, Phys. Rev. Phys. Educ. Res. 13, 020125 (2017).

[42] A. D. Smith, J. P. Mestre, and B. H. Ross, Eye-gaze patterns as students study worked-out examples in mechanics, Phys. Rev. ST Phys. Educ. Res. 6, 020118 (2010).

[43] A. M. Madsen, A. M. Larson, L. C. Loschky, and N. S. Rebello, Differences in visual attention between those who correctly and incorrectly answer physics problems, Phys. Rev. ST Phys. Educ. Res. 8, 010122 (2012).

[44] A. Madsen, A. Rouinfar, A. M. Larson, L. C. Loschky, and N.S. Rebello, Can short duration visual cues influence students' reasoning and eye movements in physics problems?, Phys. Rev. ST Phys. Educ. Res. 9, 020104 (2013).

[45] A. Rouinfar, E. Agra, A. M. Larson, N. S. Rebello, and L. C. Loschky, Linking attentional processes and conceptual problem solving: Visual cues facilitate the automaticity of extracting relevant information from diagrams, Front. Psychol. 5, 1094 (2014).
[46] M. Kekule, Students' approaches when dealing with kinematics graphs explored by eye-tracking research method, in Proceedings of the Frontiers in Mathematics and Science Education Research Conference, FISER' 2014 (Science Education research Group as Eastern Mediterranean University, Famagusta, 2014), pp. 108117; In Special issue of European Journal of Science and Mathematics Education, ISSN 2301-251X.

[47] S. C. Chen, H. C. She, M. H. Chuang, J. Y. Wu, J. L. Tsai, and T. P. Jung, Eye movements predict students' computerbased assessment performance of physics concepts in different presentation modalities, Comput. Educ. 74, 61 (2014).

[48] M. Kekule, Students' different approaches to solving problems from kinematics in respect of good and poor performance, International Conference on Contemporary Issues in Education (2015), pp. 126-134.

[49] M. Kekule, J. Viiri, J. Isoniemi, and J. Hautala, Uncovering the effect of task representation on students' problem solving by eye tracking, Proceedings of the ESERA 2017 Conference (2017), https://keynote.conference-services.net/resources/ 444/5233/pdf/ESERA2017_0116_paper.pdf.

[50] P. A. Carpenter and P. Shah, A model of the perceptual and conceptual processes in graph comprehension, J. Exp. Psychol. Appl. 4, 75 (1998).

[51] J. Goldberg and J. Helfman, Eye tracking for visualization evaluation: Reading values on linear versus radial graphs, Inf. Vis. 10, 182 (2011).

[52] P. Nieminen, A. Savinainen, and J. Viiri, Force concept inventory-based multiple-choice test for investigating students' representational consistency, Phys. Rev. ST Phys. Educ. Res. 6, 020109 (2010).

[53] See Supplemental Material at http://link.aps.org/ supplemental/10.1103/PhysRevPhysEducRes.14.020109 for the multiple-choice questions used in the study.

[54] Q. Wang, S. Yang, M. Liu, Z. Cao, and Q. Ma, An eyetracking study of website complexity from cognitive load perspective, Decision Support Systems 62, 1 (2014).

[55] K. F. Van Orden, W. Limbert, S. Makeig, and T.-P. Jung, Eye activity correlates of workload during a visuospatial memory task, Human Factors 43, 111 (2001).

[56] A. Susac, A. Bubic, J. Kaponja, M. Planinic, and M. Palmovic, Eye movements reveal students' strategies in simple equation solving, Int. J. Sci. Math. Educ. 12, 555 (2014).

[57] J. D. Bransford and D. L. Schwartz, Rethinking transfer: A simple proposal with multiple implications, Rev. Res. Educ. 24, 61 (1999). 